\title{
Shear Bond Strength of Different Adhesive Materials used for Bonding Orthodontic Brackets : A Comparative in vitro Study
}

\author{
Dr Muzib Shahi Shaik,' Dr Snigdha Pattanaik,2 Dr Sudhakar Pathuri,3 Dr Arunachalam Sivakumar4 \\ IPrivate Practitioner, Silver Smiles Super-specialty Dental Hospital, Guntur, India \\ ${ }^{2}$ Assistant Professor, Institute of Dental Sciences, Bhubaneswar, India \\ 3.4Professor, Vishnu Dental College, Bhimavaram, India
}

Correspondence: Dr Snigdha Pattanaik; Email: dr.snigdhapattanaik@gmail.com

\section{ABSTRACT}

Introduction: Bond strength is an important property and determines the amount of force delivered and treatment duration in orthodontics. Many light-cured bonding materials are being used; but it is required to determine the most efficient one with desired bond strength.

Objective: To determine and compare the shear bond strength of three visible light-cured composites (Transbond XT, Heliosit and Enlight) and two self-cured composites (Rely-a-bond and Concise).

Materials \& Method: 100 extracted premolars were collected and randomly divided into 5 test groups of different adhesives. Brackets were bonded to the teeth in each test group with the respective adhesive according to the manufacturer's instructions. Each specimen was debonded using Universal Testing Machine and the shear bond strength for each specimen was calculated. All the groups were compared by ANOVA one-way test.

Results: There were statistically significant differences among the five groups ( $P<0.05)$. The shear bond strength of Enlight (13.92 $\pm 3.92)$ is similar to Transbond XT (14.30 \pm 4.35$)$.

Conclusion: Light cure composites showed higher bond strength than self cure composites.

Keywords: adhesive material, bond failure, orthodontic bracket, shear bond strength

\section{INTRODUCTION}

Bonding of orthodontic attachments using orthodontic adhesives is one of the important procedures in clinical orthodontic practice and is the most significant development over the past three decades. Direct bonding technique has opened new horizons for researchers to introduce new adhesive materials and improved techniques.

Buonocore' in 1955 demonstrated a simple method to increase adhesion of acrylic filling material to enamel surface by acid pre-treatment with $85 \%$ phosphoric acid. Acid etch technique was originally introduced in restorative dentistry for enhancing adhesions of restorative materials to etched enamel surface. Newman ${ }^{2}$ in 1965 used this technique for bonding plastic brackets with epoxy resin. This paved the way for direct bonding of bracket in orthodontic practice. Bowen's resin or Bisphenol A glycidyl dimethacrylate (Bis-GMA) was developed by Bowen in 1962 for restorative purpose. ${ }^{3}$ Bowen's resin is an acrylic-modified epoxy resin with setting advantage of acrylic and dimensional stability and strength of epoxy resin.

As new adhesives, composite resins and newer bonding techniques were being introduced in restorative dentistry; orthodontists also adapted some of these innovations. Various modifications were made to Bowen's resin with the addition of co-monomer, variable amounts of fillers and silane coupling agent to alter their properties and enhance their performance for the clinical use. Addition of filler particles to these resins to form composites greatly increased their strength and mechanical properties.

An ideal orthodontic adhesive should have optimal bond strength when the brackets are bonded to the etched enamel surface to withstand masticatory forces and forces of the orthodontic appliance. On the other hand, it should also debond without causing any damage to the enamel at the end of the treatment. The bond strength and debonding character of Transbond XT have been extensively studied and reported to have optimal bond strength with wide clinical acceptance. ${ }^{4}$ 
Transbond $\mathrm{XT}$ is a conventional light-cured BISGMAbased (Bis Phenol A Glycidyl dimethacrylate) composite resin popularly used as orthodontic adhesive agent with optimal bond strength. ${ }^{4}$ It consists of BISGMA resin (monomer) matrix and TEGDMA (Triethylene glycal dimethacrylate) as diluents. It also contains filler particles like ground glass or silica and silane coupling agent to provide bond between inorganic and organic resin matrix. Camphoroquinone is used as a photo initiator for the polymerization of monomer.

The quest to overcome the shortcomings of conventional filled composites has led to the development of "flowable Composites". 5 Flowable composites achieved great attention due to their clinical handling characteristics like non-stickiness, fluid injectability, adequate working time and short cure time. They are especially useful during indirect bonding of attachments. Heliosit, a flowable composite although initially intended for bonding of brackets, its application as a bonding agent for bonding lingual retainers, and even as a luting cement for prosthesis has been tested. Heliosit as a bonding agent of brackets has been scarcely studied. Heliosit composition includes Bis-GMA (50-100\%), UDMA (10-25\%) and Deca Methyl endi methacrylate (10-25\%). ${ }^{4}$

Enlight composition includes Dimethacrylate monomer $20-30 \%$, silicate filler $70-80 \%$, other supplements $4 \%$, among them camphoroquinone is the activator.

Self-curing resins, for a long time were the only option for dental restorative and orthodontic bonding procedures. This kind of material boasts great clinical efficiency but its short handling time hinders the procedure. Bis-GMA or Bowen's resin is a self-cure resin introduced in late 1960's. Though it provided good bond strength, it had few inherent flaws as it was extremely technique-sensitive. The self curing composites used in this study are Concise and Rely-a-bond.

The composition of Concise includes: Enamel bond system Resin A which contains TEGDMA (40-50\%), Bis-GMA (40-50\%), 2,2P-tolylimnol diethanol (1-10\%), 2-benzotriazol4-methylphenol (1-10\%) and Hydroquinone (<0.03). Enamel bond system B includes: Filler (70-80\%), Bisphenol A diglycidyl ether dimethacrylate (10-20\%) and TEGDMA (5$10 \%)$. Rely-a-bond Paste A include: silane treated quartz (75-85\%), Bis-GMA (10-20\%), TEGDMA (1-10\%), silica (<2\%), 2-Benzotriazyl-4-methyl phenol (<1\%). Paste B include: silane treated quartz (75-85\%), Bis-GMA (10-20\%), TEGDMA (1-10\%), silica (<2\%), 2-Benzotriazyl-4-methyl phenol (<1\%).

Hence the present study attempts to determine the shear bond strength of five different adhesives and to compare between light-cured \& self-cured adhesives used in the study.

\section{MATERIALS AND METHOD}

A total of one hundred freshly extracted maxillary premolar teeth and 100 pre-adjusted edgewise upper premolar stainless steel brackets (Ormco Mini 2000 series) were used in the study. The teeth used were anatomically and morphologically sound and non-carious. Ethical clearance was obtained from the Institution for the use of natural teeth in the study.

The freshly extracted teeth were cleaned to remove blood or any tissue debris and were stored in $0.1 \%$ thymol solution to prevent bacterial contamination and dehydration. The teeth were then mounted on self-cured, acrylic blocks of dimensions $25 \times 10 \times 10 \mathrm{~mm}$ such that the roots were completely embedded into the acrylic block up to cemento-enamel junction. The buccal surface of crown was perpendicular to the base of the block. The acrylic blocks were numbered from 1 to 100 to differentiate between different groups.

The teeth samples were divided into five following groups:

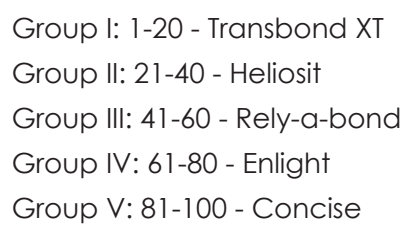

\section{Bonding procedure:}

The buccal surface of the teeth was polished with pumice slurry. After polishing, the teeth were washed with distilled water and dried. $37 \%$ ortho phosphoric acid was applied to the labial surface and left for a period of 15 seconds. The tooth was then washed and air-dried until a dull frosty appearance was seen. The procedure was done for all the test specimens to be bonded with five adhesive groups to be evaluated.

The Group I was bonded to the tooth structure using primer and adhesive of Transbond XT. Group II with Heliosit adhesive, Group III with primer-adhesive combination of Rely-A Bond, Group IV with Enlight adhesive and Group V with Concise adhesive.

The light cure adhesive was cured using a MONITEX Blue LEX GT 1200 LED (light emitting diode) curing unit. The adhesive was cured from the occlusal, gingival, mesial and distal aspects for 10 seconds each. The bonded teeth were then kept in distilled water at room temperature for 24 hours before debonding.

\section{Evaluation of bond strength:}

Debonding was carried out using LLOYD Universal Testing Machine LR 50K (Figure 1, 2). The LLOYD unit was attached to an electronic console that displayed the debonding forces acting between the jaws. Thus the exact force at which the bracket debonded was noted from the 


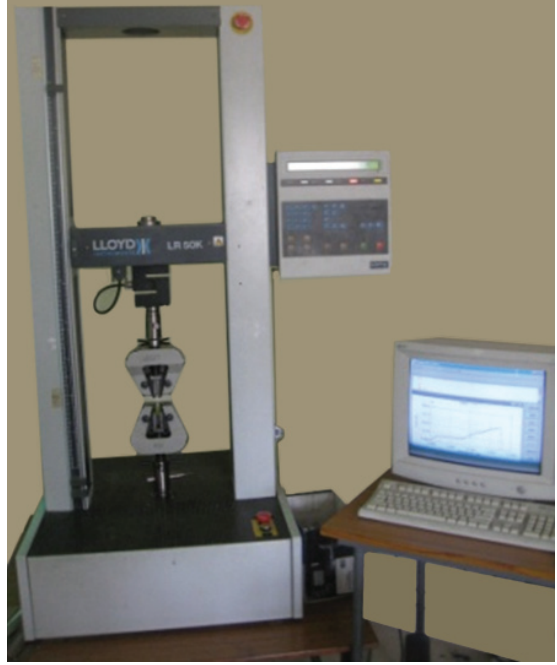

Figure 1: Universal Testing Machine (LLOYD LR 50K)

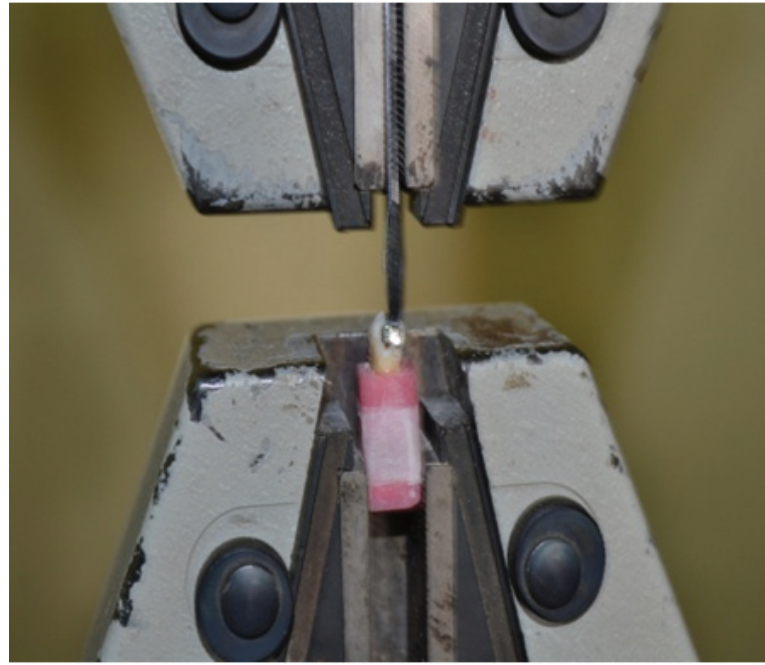

Figure 2: Measuring shear bond strength under Universal Testing Machine console. This force was expressed in Newtons. To evaluate the shear bond strength in MPa from the force value, the following formula was used:

Shear Bond Strength (MPa) $=\frac{\text { Force in Newtons }}{\text { Area of the bracket base }(\mathrm{sq} \mathrm{mm})}$

The area of the bracket base was measured as $9.63 \mathrm{~mm}^{2}$ by using digital Vernier calipers.

\section{RESULT}

The result of the shear bond strength of the five adhesives: Group I- Transbond XT, Group II-Heliosit, Group III- Rely-abond, Group IV- Enlight and Group V- Concise with their interpretation are presented in Table 1 and Table 2, and graphically represented in Graph 1.

Descriptive statistics like mean and standard deviation were calculated for every group. Group differences were analyzed with one-way analysis of variance (ANOVA) (Table 2).

\section{DISCUSSION}

Manufacturers are continuously introducing new adhesive systems to the dental profession that are claimed to be more reliable, stronger, adhere better to enamel and dentin, less liable to leak at the margins and easy to handle.

In the present study five different adhesive materials: Transbond XT, Heliosit, Rely-a-bond, Enlight and Concise were assessed. The Light cure composites used in the present study were Transbond XT, Heliosit and Enlight. Transbond XT is a commercially available (3M Unitek, California) light-cured Bis-GMA based composite resin and is a popularly used orthodontic adhesive agent. It has been extensively evaluated for its bond strength and found to have optimal bond strength.

In the present study mean shear bond strength of Transbond XT achieved was 14.30 MPa \pm 4.35. The shear bond strength of Transbond XT reported by Durrani et al was 25.5 MPa $\pm 1.69 \mathrm{MPa}$. This was higher than the previous studies but was comparable to the studies of Tecco et $a^{6}$

Table 1: Descriptive statistics for shear bond strength of five groups

\begin{tabular}{|l|c|c|c|c|}
\hline \multicolumn{1}{|c|}{ Group } & $\begin{array}{c}\text { No. of } \\
\text { samples }\end{array}$ & $\begin{array}{c}\text { Mean Shear Bond } \\
\text { Strength (MPa) }\end{array}$ & $\begin{array}{c}\text { Standard } \\
\text { Deviation }\end{array}$ & $\begin{array}{c}\text { Lower value } \\
\text { Upper value }\end{array}$ \\
\hline Group I: Transbond XT & $\mathbf{2 0}$ & 14.30 & 4.35 & 21.32 \\
\hline Group II: Heliosit & $\mathbf{2 0}$ & 11.46 & 3.87 & 20.05 \\
\hline Group III: Rely-a-bond & $\mathbf{2 0}$ & 6.78 & 1.83 & 4.32 \\
\hline Group IV: Enlight & $\mathbf{2 0}$ & 13.92 & 3.92 & 23.36 \\
\hline Group V: Concise & $\mathbf{2 0}$ & 10.78 & 4.16 & 21.22 \\
\hline
\end{tabular}


Table 2: Analysis of variance for shear bond strength of five groups

\begin{tabular}{|l|c|c|c|c|c|}
\hline $\begin{array}{c}\text { Source of } \\
\text { Variation }\end{array}$ & $\begin{array}{c}\text { Degree of } \\
\text { freedom }\end{array}$ & $\begin{array}{c}\text { Sum of } \\
\text { Squares }\end{array}$ & $\begin{array}{c}\text { Mean sum of } \\
\text { squares }\end{array}$ & $\begin{array}{c}\text { Variance ration } \\
\text { F-value }\end{array}$ & p-value \\
\hline Between Groups & 4 & 729.626 & 182.406 & 13.044 & $0.000 *$ \\
\hline Within Groups & 95 & $1,328.486$ & 13.984 & & \\
\hline Total & 99 & $2,058.111$ & & \\
\hline
\end{tabular}

*Highly significant $p<0.05$

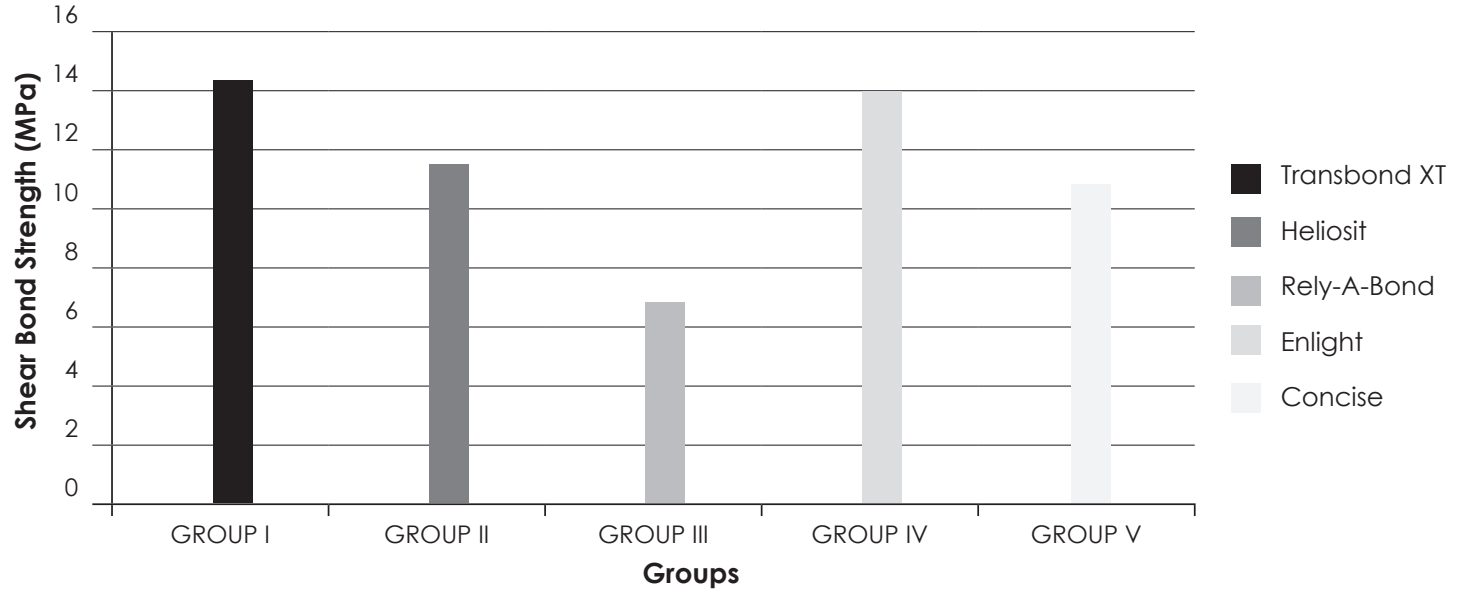

Graph 1: Mean shear bond strength of different groups

(23.23 MPa \pm 5.23 MPa), Atillio et $\mathrm{al}^{7}$ (23.47 MPa \pm 4.86 MPa), Rock and Abdullah ${ }^{8}$ (8-23 MPa), Sinha et al ${ }^{9}$ (18.9 MPa), Sunna et $a l^{10}$ (11-22 MPa) and Rix et $a^{11}$ (20.19 MPa).

In the present study, when the shear bond strength of Transbond XT and Heliosit were compared; Transbond XT showed a mean of $14.30 \pm 4.35 \mathrm{MPa}$ and Heliosit showed $11.4 \pm 3.87 \mathrm{MPa}$. Durrani compared the shear bond strength of Heliosit with that of Transbond XT; the bond strength of Heliosit was $10.54 \pm 1.86 \mathrm{MPa}$. This bond strength is higher than the reports of Bradburn and Pender ${ }^{12}$ (7.22 MPa $\pm 2.11 \mathrm{MPa}$ ), but considerably less than those achieved by Joseph and Rossouw ${ }^{13}(17.80 \mathrm{MPa} \pm 3.54 \mathrm{MPa})$ and Schmidlin et $\mathrm{al}^{14}(16.6 \mathrm{MPa} \pm 6.4 \mathrm{MPa})$.

When Transbond XT and Enlight were compared; there was no significant difference in shear bond strength between them. The mean shear bond strengths of Transbond XT and Enlight were 14.30 $\pm 4.35 \mathrm{MPa}$ and 13.92 $\pm 3.92 \mathrm{MPa}$ respectively. Owens reported the shear bond strength of Transbond XT and Enlight as $7.9 \pm 2.1$ and 6.8 \pm 2.1 respectively. ${ }^{5}$ Linn et al reported the shear bond strength of Transbond XT and Enlight as $16.27 \pm 4.74$ and $14.76 \pm 4.0$ respectively. ${ }^{15}$ Banerjee and Sable compared the shear bond strength of Enlight (Ormco Corporation) and Transbond XT (3M Unitek) when cured with LED light. Transbond XT showed the bond strength of $14.6 \mathrm{MPa}$ and
Enlight showed $13.50 \mathrm{MPa}^{16}$

The self curing composites used in this study were Concise and Rely-a-bond. When Transbond XT and Concise were compared; the shear bond strength obtained were $14.30 \pm$ $4.35 \mathrm{MPa}$ and $10.78 \pm 4.16 \mathrm{MPa}$ respectively. Rastelli et $\mathrm{al}^{17}$ compared two fluoride-releasing composite resins (Ultra bond and Rely-a-bond) and a conventional composite resin (Concise). The result showed that the three groups were significantly different from one another. Concise had the highest mean shear bond strength $(24.54 \pm 6.98 \mathrm{MPa})$ compared to other groups. These findings corroborated the work of Murray et $\mathrm{al}^{18}$ and Reicheneder et $\mathrm{al} ;{ }^{19}$ they also found higher shear bond strength values when using Concise.

Concise exhibited the highest shear bond strength due to its high filler content since the content of inorganic particles directly influence the resistance of composite resin. Ahmed found shear bond strength of Transbond XT and Concise were 10.537 Mpa and 10.970 MPa respectively. ${ }^{20}$ The lower shear bond obtained in the present study may be due to the technique sensitivity involved during the mixing.

In the present study Rely-a-bond showed mean shear bond strength of $6.78 \pm 1.83 \mathrm{MPa}$. Similar study conducted by Rastelli demonstrated the strength of $16.46 \pm 5.72 \mathrm{MPa}^{17}$ 
The findings were similar to those obtained by Sinha et al (19.0 MPa). ${ }^{9}$ Komori and Ishikawa however found a different result for the same self-curing resin $(25.7 \pm 3.6$ MPa). ${ }^{21}$ These differences might be due to the thermocycled samples at various test temperatures.

There were few limitations in the present in vitro study. Efforts were made to replicate the oral environment however the oral environment cannot be simulated outside the mouth. It is due to the bio-degradation in the oral cavity as a result of the combination of disintegration and dissolution in saliva, chemical and physical degradation, wear caused by chewing food, erosion by the food consumed, and bacterial activity. Thus it is such a complex interaction of processes that cannot be simulated in vitro. More study is warranted in this subject as there have been conflicting reports in the literature.

\section{CONCLUSION}

It is evident from the present investigation that; the composite resin adhesives should be utilized in orthodontics for their good shear bond strength.

- Acceptable shear bond strength was achieved by Transbond XT, Heliosit, Rely-a-bond, Concise adhesives.

- Enlight has shear bond strength similar to Transbond XT, hence it can be used as an alternative to Transbond XT for bonding orthodontic brackets.

- $\quad$ Light-cure composite has higher shear bond strength than self-cure composites. Hence, Light cure composites are preferred over self cured composites.

\section{REFERENCES}

1. Buonocore MG. A simple method of increasing the adhesion of acrylic filling materials to enamel surface. J Dent Res.1955; 34 : $849-853$.

2. Newman GV. Epoxy adhesives for orthodontic attachments: progress report. Am J Orthod Dentofacial Orthop.1965; 51: $901-912$.

3. Bowen RL. Use of epoxy resin in restorative material. J Dent Res 1956; 35:360-369.

4. Zachrisson B. Bonding in orthodontics. Graber T, Vanarsdall R. eds. Orthodontics Current Principles and Techniques. St Louis, Mo: MosbyYearbook, Inc., 1994; 542-559.

5. Owens SE Jr, Miller BH. A Comparison of Shear Bond Strengths of Three Visible Light-Cured Orthodontic Adhesives Angle Orthod. $2000 ; 70$.

6. Tecco S, Traini T, Caputi S, Festa F, deLuca V, D'Attilio M. A new one-step dental flowable composite for orthodontic use: An in vitro bond strength study. Angle Orthod. 2005; 75: 672-7.

7. Attilio DM, Traini T, Di lorio D, Varvara G, Festa F, Tecco S. Shear bond strength, bond failure, and scanning electron microscopy analysis of a new flowable composite for orthodontic use. Angle Orthod. 2005; 75: 410-5.

8. Rock WP, Abdullah MS. Shear bond strengths produced by composite and compomer light cured orthodontic adhesives. J Dent. 1997; 25: 243-9

9. Sinha PK, Nanda RS, Duncanson MG Jr, Hosier MJ. In vitro evaluation of matrix-bound fluoride-releasing orthodontic bonding adhesives. Am J Orthod Dentofacial Orthop. 1997; 111:276-82.

10. Sunna S, Rock WP. An ex vivo investigation into the bond strength of orthodontic brackets and adhesive systems. Br J Orthod. $1999 ; 26$ : 47-50

11. Rix D, Foley TF, Mamandras A. Comparison of bond strength of three adhesives: composite resin, hybrid GIC, and glass filled GIC. Am J Orthod Dentofacial Orthop. 2001; 119:36-42.

12. Bradburn G, Pender N. An in vitro study of the bond strength of two light-cured composites used in the direct bonding of orthodontic brackets to molars. Am J Orthod Dentofacial Orthop. 1992; 102: 418-26.

13. Joseph VP, Rossouw E. The shear bond strengths of stainless steel and ceramic brackets used with chemically and light activated composite resins. Am J Orthod Dentofacial Orthop. 1990; 97: 121-5.

14. Schmidlin PR, Schatzle M, Fischer J, Attin T. Bonding of brackets using a caries-protective adhesive patch. J Dent. 2007.

15. Linn BJ, Berzins DW, Dhuru VB, Bradley TG. A Comparison of bond strength between direct-and indirect-bonding methods. Angle Orthod. 2006; 76:289-294.

16. Banerjee S, Sable RB. Shear Bond Strength Of Orthodontic Bonding Agents. Journal of Clinical and Diagnostic Research. 2010; 4:21 162124.

17. Rastelli MC, Coelho U, Enrique E, Jimenez O. Evaluation of shear bond strength of brackets bonded with orthodontic fluoride-releasing composite resins. Dental Press J Orthod, 2010; 15(3):106-13.

18. Murray SD, Hobson RS. Comparison of in vivo and in vitro shear bond strength. Am J Orthod Dentofacial Orthop. 2003; $123: 2-9$.

19. Reicheneder CA, Gedrange T, Lange A, Baumert U, Proffe P. Shear and tensile bond strength comparison of various contemporary orthodontic adhesive systems: An in-vitro study. Am J Orthod Dentofacial Orthop. 2009; 135:422.e 1-422.e6.

20. Ahmed MK. Evaluation of the shear bond strength of four orthodontic adhesive systems. Al-Rafidain Dent J. 2007; 7(1): 66-70.

21. Komori A, Ishikawa H. Evaluation of a resin-reinforced glass ionomer cement for use as an orthodontic bonding agent. Angle Orthod. 1997; 67(3):189-96. 